\title{
AK Sco, first detection of a highly disturbed atmosphere in a pre-main sequence close binary
}

\author{
Ana I. Gómez de Castro \\ S.D. Astronomia y Geodesia, Fac. de CC Matemáticas, Universidad Complutense de \\ Madris, E-28040 Madrid, Spain \\ aig@mat.ucm.es
}

\begin{abstract}
AK Sco is a unique source: a $~ 10$ Myrs old pre-main sequence spectroscopic binary composed of two nearly equal F5 stars that at periastron are separated by barely eleven stellar radii so, the stellar magnetospheres fill the Roche lobe at periastron. The orbit is not yet circularized $(\mathrm{e}=0.47)$ and very strong tides are expected. This makes of AK Sco, the ideal laboratory to study the effect of gravitational tides in the stellar magnetic field building up during pre-main sequence (PMS) evolution. In this letter, the detection of a highly disturbed $\left(\sigma \simeq 100 \mathrm{~km} \mathrm{~s}^{-1}\right)$ and very dense atmosphere $\left(\mathrm{n}_{\mathrm{e}}=1.6 \times 10^{10} \mathrm{~cm}^{-3}\right)$ is reported. Significant line broadening blurs any signs of ion belts or bow shocks in the spectrum of the atmospheric plasma. The radiative loses cannot be accounted solely by the dissipation of energy from the tidal wave propagating in the stellar atmosphere; neither by the accreting material. The release of internal energy from the star seems to be the most likely source of the plasma heating. This is the first clear indication of a highly disturbed atmosphere surrounding a pre-main sequence close binary.
\end{abstract}

Subject headings: stars: pre-main sequence, stars: magnetic fields, binaries: spectroscopic

\section{Introduction}

Pre-main sequence (PMS) stars are surrounded by very powerful magnetospheres that extend 2-4 stellar radii to the limits of the inner disk boundary. The magnetospheres are powered by the stellar magnetic field, the accreting infalling material and the shearing at the boundary between the magnetosphere and the inner disk (see Gómez de Castro 2009 
for a recent review). Magnetospheric heating processes during pre-main sequence evolution are poorly studied, as well as the relative relevance of the contributions mentioned above. This is partially caused by the similar densities $\left(10^{9}-10^{11} \mathrm{~cm}^{-3}\right)$ and temperatures $\left(10^{4.5}-\right.$ $10^{4.8} \mathrm{~K}$ ) of several distinct physical components in the circumstellar environment (the diskstar shear layer, the outflow and the accretion flow). Moreover, the high temperature of the plasma makes thermal broadening very large $\left(\sim 70 \mathrm{~km} \mathrm{~s}^{-1}\right)$ leaving ultraviolet high spectral resolution monitoring as the only technique to resolve the various contributions since variability time scales are expected to be very different for the various components (see i.e. Gómez de Castro \& Verdugo 2007).

Close pre-main sequence binaries are ideal targets to unveil some of the magnetospheric properties. Circumstellar accretion disks are difficult to accommodate in these systems since the strong gravitational tides break them into rings. The inner boundary of the circumbinary disk is pushed outwards by the binary orbit thus the magnetosphere of each individual star is not locked to the disk rather, it is expected that a strong coupling between the gravitational tides and the stellar magnetic fields ought to be a major source of atmospheric/magnetospheric heating. The investigation of the atmospheric properties of close pre-main sequence stars provides important clues regarding the possible role of magnetospheric dynamos in pre-main sequence evolution.

AK Sco is a unique target for this purpose; it is the only pre-main sequence binary known to date with small eccentricity and composed of two nearly equal stars that get as close as $11 R_{*}$ at periastron passage. No signs of enhanced accretion have been detected at periastron (Andersen et al. 1989, Alencar et al. 2003). Polarimetric observations have reported the largest variations of percent polarization and position angle detected in a premain sequence binary (Manset et al. 2005). Variations are found to be periodical with period equal to the gravitational tide period (half the orbital period). The main parameters of the binary are summarized in Table 1. In this letter, the profiles of the (semiforbidden) intercombination lines of the optically thin magnetospheric tracers: the CIII] at $1908 \AA$ and the Si III] at $1892 \AA$ spectral lines, are analyzed. From the analysis, it is concluded that there is an extended region of radius $\sim 1.3-4.9 \mathrm{R}_{*}$ around each star containing hot $\left(\log T_{\mathrm{e}}=4.8\right)$ and highly perturbed gas that produces a gaussian-like profile broadening with $\sigma \sim 100 \mathrm{~km} \mathrm{~s}^{-1}$. Although this broadening is highly suprathermal it could be sufalvénic if AK Sco's magnetic field is similar or higher than that reported for other pre-main sequence stars (see i.e. Johns-Krull, 2007). 


\section{Observations and measurements}

AK Sco was observed with the Space Telescope Imaging Spectrograph (STIS) on March 10, 2001. The observations were carried out with grating G230M to target the semiforbidden lines of Si III] at $1892 \AA$ and C III] at $1908 \AA$. The spectra were obtained at phase 0.2814 according to Andersen et al (1989) ephemeris (see Table 1). At this phase, the radial velocity of both components is the same thus the profiles represent the co-added contribution of both components in the same velocity space. As all indications are that the two components are equal in all respects, including spectral type, the contribution from both stars is expected to be the same.

AK Sco displays strong and very broad Si III] and C III] profiles. The observed line profiles have been converted to velocity distribution using the Doppler shift formula. Data have been processed with the Routine Science Data Pipeline (RSDP). The major source of inaccuracy in the calibration is the centering of the target in the aperture that can account for as much as $2.8 \mathrm{~km} \mathrm{~s}^{-1}$. The exposure duration was $1944 \mathrm{~s}$ that were splitted into three subexposures; the average value for each pixel and the deviation from this average are plotted in Fig. 1. A curve fit to a single gaussian plus a constant is also shown. The dispersion of the gaussians are $\sigma(\mathrm{C} \mathrm{III]})=109.5 \mathrm{~km} / \mathrm{s}$ and $\sigma(\mathrm{Si}$ III $])=106.8 \mathrm{~km} / \mathrm{s}$. The value of $\chi^{2} / \nu$ is 3.6 and 1.3 for this best gaussian fit to the Si III] and C III] lines, respectively. The instrumental profile of HST/STIS with the grating G230M has a FWHM of $30 \mathrm{~km} / \mathrm{s}$ and the stellar rotation velocity is $v \sin i=18.5 \mathrm{~km} \mathrm{~s}^{-1}$, thus line broadening 1 is more than one order of magnitude larger than expected from the rotational broadening of the atmospheric lines.

The lines fluxes are: $\mathrm{F}(\mathrm{Si}$ III $])=(4.2 \pm 0.1) \times 10^{-13} \mathrm{erg} / \mathrm{s} / \mathrm{cm}^{2}$ and $\left.\mathrm{F}(\mathrm{C} \mathrm{III}]\right)=(2.9 \pm$ $0.1) \times 10^{-13} \mathrm{erg} / \mathrm{s} / \mathrm{cm}^{2}$, after correction for extinction using Valencic et al. $2004 \mathrm{UV}$ law $(\mathrm{R}=$ $\mathrm{A}_{V} / \mathrm{E}_{B-V}=4.3$ from Manset et al. 2005 and $\mathrm{A}_{V}=0.5 \pm 0.1 \mathrm{mag}$ from Alencar et al. 2003). The lines ratio is calculated to be $\mathrm{F}(\mathrm{Si} \mathrm{III}]) / \mathrm{F}(\mathrm{C}$ III $])=1.4 \pm 0.2$. This value is similar to that derived from earlier (1982 and 1988) low resolution observations obtained with the International Ultraviolet Explorer (IUE) (Gómez de Castro \& Franqueira 1997). From the IUE low dispersion spectra a C IV $(\lambda \lambda 1548,1550 \AA)$ flux of $(1.6 \pm 0.1) \times 10^{-12} \mathrm{erg} / \mathrm{s} / \mathrm{cm}^{2}$ and a ratio $\mathrm{F}(\mathrm{C}$ IV) $/ \mathrm{F}(\mathrm{Si}$ III]) of $1.9 \pm 0.4$ was derived. There are only three low resolution observations carried out with the IUE, two of them during the same cycle at periastron (phase $=0.123$ ) and at apastron (phase $=0.708$ ). No significant variations in the C III], Si III] and C IV fluxes 2 are found though the IUE fluxes are about a factor of $\simeq 2$ higher than that

\footnotetext{
${ }^{1}$ The FWHM of the profiles is $\left.\mathrm{FWHM}(\mathrm{Si} \mathrm{III}]\right)=251 \mathrm{~km} / \mathrm{s}$ and $\left.\mathrm{FWHM}(\mathrm{C} \mathrm{III}]\right)=258 \mathrm{~km} / \mathrm{s}$.

${ }^{2}$ There are however, variations by a factor of $\sim 2$ in the O I $(\lambda 1304 \AA)$ and $\mathrm{C}$ II resonance multiplet at $1335 \AA$; these lines trace cooler and lower density plasma.
} 
measured with the HST.

\section{Constraints from the lines flux}

Assuming collisional equilibrium, the $\mathrm{F}(\mathrm{Si} \mathrm{III}]) / \mathrm{F}(\mathrm{C}$ III]) and $\mathrm{F}(\mathrm{C}$ IV)/F(Si III]) ratios indicate that the physical conditions in this Hot Line Emission Region (HLER) are $\log n_{e}\left(\mathrm{~cm}^{-3}\right)=10.2$ and $\log T_{e}(\mathrm{~K})=4.8$. An optically thin collisional plasma with these properties radiates $2.4 \times 10^{-3}$ of the total flux in the C III] line; thus the luminosity of the HLER is $3.1 \times 10^{32} \mathrm{erg} / \mathrm{s}$ or $0.08 L_{\odot}\left(0.04 L_{\odot}\right.$ per star assuming the same contribution per component); a distance, $d$, of 145 pc to AK Sco has been assumed. To account for this high luminosity an accretion rate 3 of $\dot{M}_{a}=0.9 \times 10^{-7} \mathrm{M}_{\odot} \mathrm{yr}^{-1}$ is required. The viscous release of this gravitational energy in the circumbinary disk would affect significantly the infrared spectral energy distribution (SED) however, good fits are obtained with passive disk models suggesting that the accretion rate is not very much above $1 \times 10^{-8} \mathrm{M}_{\odot} \mathrm{yr}^{-1}$ (see i.e. Alencar et al. 2003 for a discussion on the SED fit).

The characteristic size of the HLER, $R_{e}$, is given by,

$$
f\left[\left(\frac{R_{e}}{R_{*}}\right)^{3}-1\right]=\frac{3 F_{l} d^{2}}{<j_{l}>R_{*}^{3}}=0.4
$$

where $R_{e}$ is the radius of an equivalent homogeneous spherical envelope around each star, with $\left\langle j_{l}\right\rangle$ representing the average emissivity in the (Si III]) lines formation region, which for this study, has been assumed to be of the order of $j_{l}=2.38 \times 10^{-4} \mathrm{erg} \mathrm{s}^{-1} \mathrm{~cm}^{-3}$ using the atomic parameters from the Chianti Atomic Data Base 4 ). Here $f$ represents the volume filling factor of the radiating plasma. For $f \sim 0.1, R_{e}$ reaches $1.7 \mathrm{R}_{*}$. This is an indication of the HLER being significantly more extended than the atmosphere of a cool main sequence star.

\section{Constraints from the line broadening}

Line broadening is highly suprathermal (thermal broadening at $10^{4.8} \mathrm{~K}$ generates a FWHM of $73 \mathrm{~km} / \mathrm{s}$, a factor of $\sim 3$ smaller than the observed). In principle, such a broad ki-

\footnotetext{
${ }^{3} \mathrm{~A}$ radius of $0.43 \mathrm{AU}$ (three times the semimajor axis of the orbit) is estimated for the gap of the circumbinary accretion disk based on numerical simulations of the evolution of binary systems (Artymovicz \& Lubow, 1997).

${ }^{4}$ URL:www.damtp.cam.ac.uk/user/astro/chianti/
} 
netic velocity distribution of the emitting ions could be produced, as in the Sun, by resonances between Alfvén waves propagating in the atmosphere and the giro-frequency of the ions, driving the Si III] and C III] ions to the very high kinetic temperature of $T_{\text {kin }}=0.75 \times 10^{6} \mathrm{~K}$ (see i.e. Cranmer 2002). However, the strength of this broad component must correlate with the coronal X-ray surface flux produced by the bremsstrahlung radiation of the high velocity electrons, as shown for magnetically active stars by Wood et al. 1997. Though AK Sco X-ray surface flux, $F_{X}=6.49 \times 10^{5} \mathrm{erg} / \mathrm{s} / \mathrm{cm}^{2}$ (derived from Steltzer et al. 2006 based on CHANDRA observations) is similar to the detected in class IV-V F5 stars (see Ayres et al. 1995), the CIV flux exceeds by $\sim 4.5$ orders of magnitude the flux predicted by the active stars CIV-X ray surface flux correlation. Thus, the HLER radiation cannot be accounted for solely by this mechanism.

The high symmetry of the profiles is suggestive of them being formed in a rotating ionized ring or belt such as the observed around RW Aur (Gómez de Castro \& Verdugo 2003). However the characteristics of AK Sco orbit imposes severe constraints on the possible location of the material with Keplerian-like orbits. On the one hand, circumstellar material is forced to be between the stellar corona $\left(\sim 2 R_{*}\right)$ and the location of the Lagrange point at periastron $\left(5.5 R_{*}\right)$. Plasma in such a hypothetical ring systems surrounding each star would be orbiting with velocities in the range $180<V_{\text {ring }}<280 \mathrm{~km} \mathrm{~s}^{-1}$ and thus, could only contribute to the line wings. On the other hand, the binary cleans the inner region of the circumbinary disk to about 0.43 AU thus, any ionized material in this boundary would be rotating at Keplerian velocities $\sim 75 \mathrm{~km} \mathrm{~s}^{-1}$. As a consequence, the profile broadening can neither be accounted for by rotating rings around the stars nor by a circumbinary belt. Moreover the low rotation velocity of the stars precludes that line broadening can be accounted by an extended magnetosphere, rigid-body like rotation as shown in Fig 2 . Line profiles have been simulated for various emissivity and density profiles for a corotating magnetospheric shell and belt. The energy radiated in the lines per unit volume, $\epsilon_{\text {rad,l }}$, at a given distance, $r$, from the center of the star is given to first order as: $\epsilon_{r a d, l}=j(r) \times \rho(r) \propto r^{\alpha}$ where $j(r)$ represents the line emissivity and $\rho$ the density of emitting ions. As shown in Fig 2 , simple spherical and cylindrical distributions (simulating a shell-like or a belt-like structure, respectively) cannot account for the observed widths of the HLER lines. The optimal fit is obtained by adding a Maxwellian velocity distribution with variance from $\sigma=74 \mathrm{~km} / \mathrm{s}$ for a corotating ring peaking at $\mathrm{d}=5.5 R_{*}\left(\chi^{2}=27.5\right.$ and $\left.\chi^{2} / \nu=1.44\right)$ to $\sigma=88 \mathrm{~km} / \mathrm{s}$ for a spherical envelope with $\epsilon_{\text {rad }} \propto r^{-2}\left(\chi^{2}=19.5\right.$ and $\left.\chi^{2} / \nu=1.2\right)$. In all cases, the Maxwellian velocity field is highly supersonic and blurs the possible kinematical signatures of rotating structures around the star. 


\section{Conclusions: on the source of the HLER heating}

AK Sco has an UV excess that accounts by as much as $0.04 \mathrm{~L}_{\odot}$ per component. This energy cannot be fed into the system solely by the gravitational energy lost by the accretion flow channelled by the surrounding disk since the accretion rates required are about one order of magnitude larger than that derived from the infrared properties of the disk. Even if the contribution of the fresh deuterium to the nuclear reactions is taken into account (Siess et al 1997), the accretion rate is a factor of ten smaller than that required to produce the observed luminosity 5 .

The gravitational energy stored in the epicyclic motion of the relative orbit is $\epsilon_{o}=$ $\frac{1}{2} \frac{M_{1} M_{2}}{M_{1}+M_{2}} e^{2} \omega^{2} a^{2}=2.36 \times 10^{46} \mathrm{erg}$ where $M_{1}$ and $M_{2}$ are the masses of the two components of the system, $e$ the eccentricity, $a$ the semimajor axis and $\omega=\frac{2 \pi}{P}$ with $P$ the period. If the gravitational energy were dissipated through the HLER radiation, it would be damped in a time scale of: $\tau_{\text {tide }}=\epsilon_{o} / 0.08 L_{\odot}=2.4 \times 10^{6} \mathrm{yr}^{-1}$ which is rather close to the age of AK Sco derived from the evolutionary tracks and the system should be already circularized. Henceforth, an additional source of energy needs to be hypothesized to power the HLER. This source also, should be acting in other pre-main sequence stars and be partially responsible of the observed UV excess.

Finally, notice that the magnetic diffusivity in the HLER, $\eta$, is very small (just 0.0045 $\mathrm{cm}^{2} / \mathrm{s}$ ) leading to very high Reynolds magnetic numbers and magnetic flux freezing. This has two important consequences: (1) the tidal deformation wave induces magnetic multipoles into the stellar dipolar fields that can be responsible of the unusually strong periodic polarimetric variations of the system and (2) the Alfvén waves induced by the tide will have very large damping lengths due to magnetic resistivity effects. The non-linear wave-wave interaction will lead to a turbulent cascade that will end at a scale determined by the dominant damping mechanism. For the HLER physical properties, this will be collisions between the ionized HLER material and the neutral infalling gas from the accretion flow that, in turn, strongly depends on the ill determined relative densities of these two components. If the observed broadening of the HLER lines is interpreted as a sign of magnetohydrodynamical turbulence this would imply that the inferred mean-field strength of the turbulent component is $3.5-$ 4.6G. This value is reasonable for the magnetosphere of a T Tauri star where typical surface

\footnotetext{
${ }^{5}$ An estimate of the luminosity excess caused by accreting fresh deuterium can be made from the nuclear energy released per reaction is $Q_{D}=5.5 \mathrm{MeV}$. The luminosity released is given by: $L_{D}=$ $(1 / 2)\left(m_{D} / m\right) n_{A} \dot{M}_{a} Q_{D}$ where $m_{D} / m$ is the mass fraction of deuterium in the accreting matter, $n_{A}$ the Avogadro number and the factor of $(1 / 2)$ is introduced to account for the Deuterium mass number. Assuming a mass fraction deuterium to hydrogen of $10^{-5}$, solar abundances and an accretion rate of $1.0 \times 10^{-8} \mathrm{M}_{\odot} \mathrm{yr}^{-1}$, the luminosity excess will be $0.004 \mathrm{~L}_{\odot}$.
} 
fields are $0.3-1 \mathrm{kG}$ (Johns-Krull, 2007). However, this value would imply that the $\beta$ of the atmospheric plasma is as high as 0.14 !.

To conclude, the observations presented in this letter indicate that AK Sco is the first pre-main sequence binary for which evidence of a highly perturbed atmosphere has been reported. However, conclusive evidence on the source of the perturbation and the mechanism that drives it, cannot be inferred solely from these data. Further UV observations and monitorings are required.

This work has been partly financed by the Ministry of Education of Spain through grant AYA2007-67726 and by the Comunidad Autónoma de Madrid by grant CAM-S0505/ESP/0237.

HST (STIS)

\section{REFERENCES}

Artymovicz, P., Lubow, S.H., 1997, ApJ, 467, L77

Alencar,, S.H.P., Melo, C.H.F., Dullemond, C.P., Andersen, J., Batalha, C., Vaz, L.P.R., Mathieu, R.D., 2003, A\&A, 409, 1037

Andersen, J., Lindgren, H., Hazen, M.L., Mayor, M., 1989, A\&A, 219, 142

Ayres et al 1995, ApJS, 96, 223

Cranmer, S.R., 2000, ApJ, 532, 1197

Gómez de Castro, A.I. \& Franqueira, M., 1997, IUE-ULDA Guide n.8 to T Tauri Stars, ESA Scientific Publication, ESA-SP 1205.

Gómez de Castro A.I. \& Verdugo E., 2003, ApJ, 597, 443.

Gómez de Castro A.I. \& Verdugo E., 2007, ApJ, 654, L91

Gómez de Castro et al 2009, Astrophysics and Space Science, 320, 97.

Johns-Krull, C.F., 2007, ApJ, 664, 975

Manset, N., Bastien, P., Bertout, C., 2005, AJ, 129, 480

Siess, L., Forestini, M., Bertout, C., 1997, A\&A, 326, 1001 
Stelzer, B., Micela, G., Hamaguchi, K., Schmitt, J.H.M.M., 2006, A\&A, 457, 223

Valencic et al 2004, ApJ, 616, 912

Wood, B.A., Linsky, J.L., Ayres, T.R.,1997, ApJ, 478, 745 


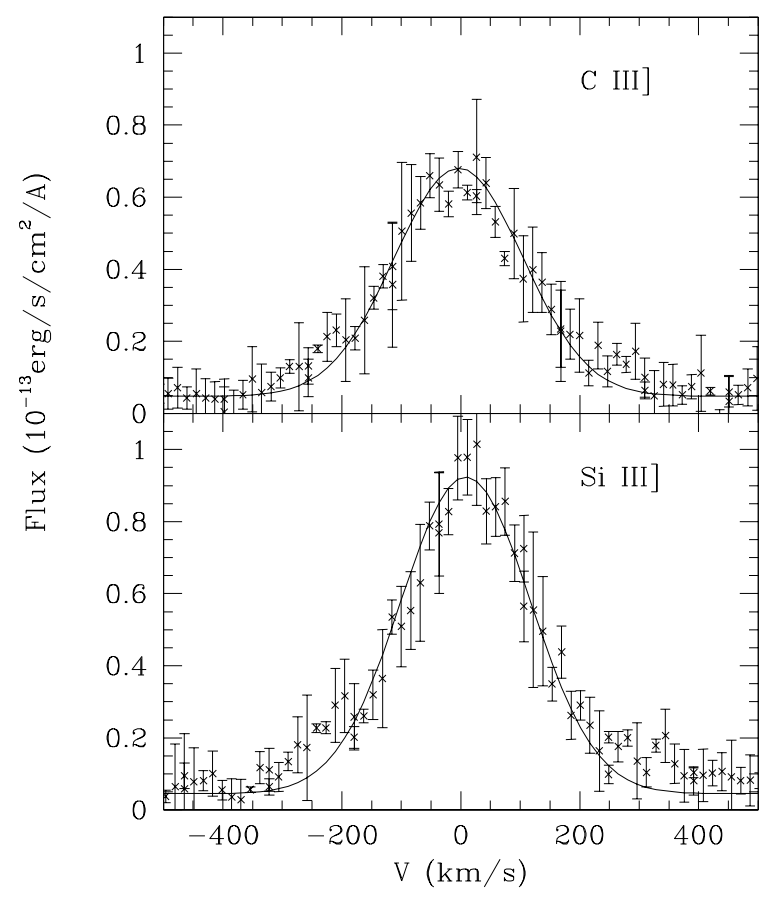

Fig. 1.- C III] (top) and Si III] (bottom) profiles of AK Sco obtained with the Hubble Space Telescope and the Space Telescope Imaging Spectrograph. Data have been processed with the Routine Science Data Pipeline (RSDP). The major source of inaccuracy in the calibration is the centering of the target in the aperture that can account for as much as $2.8 \mathrm{~km} \mathrm{~s}^{-1}$. The exposure duration was $1944 \mathrm{~s}$ that were splitted into three subexposures; the average value for each pixel and the deviation from this average (error-bar) are plotted. A curve fit to a single gaussian plus a constant is also shown. 

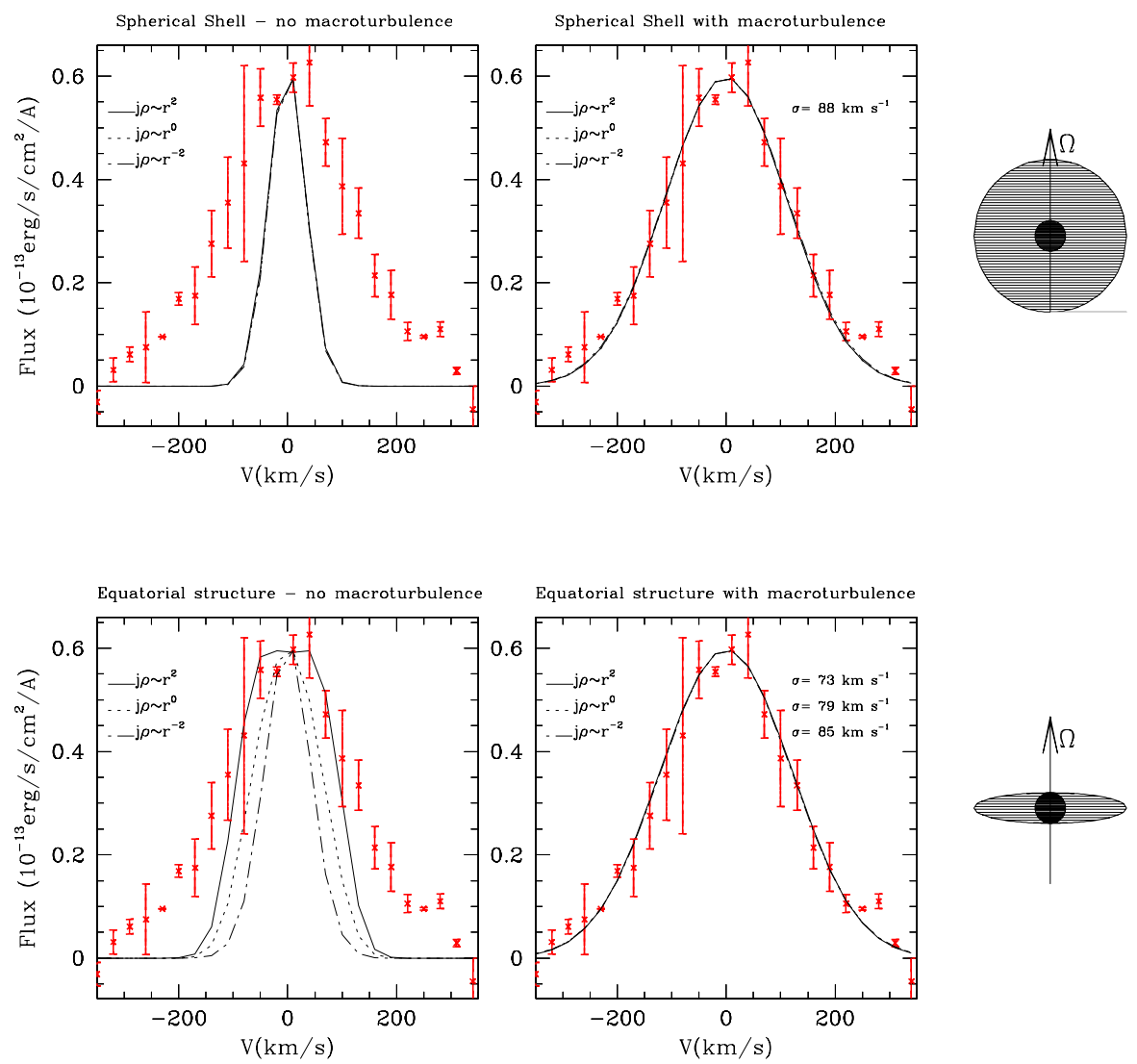

Fig. 2.- Predicted C III] line profiles for equatorial and spherical distributions of the emitting plasma. Top and bottom panels represent synthetic profiles for spherical-like and disk-like mass distributions. Three types of dependences of the line emissivity with the radius have been computed for the two possible mass distributions: $\epsilon_{r a d, l} \propto r^{\alpha}$ with $\alpha=-2,0$ and 2 , as indicated in the figures. The synthetic profiles are represented in the left panels. It is necessary to add a macro turbulence field to fit the profiles, as shown in the right panels. 
Table 1: Main properties of AK Sco spectroscopic binary system ${ }^{\mathrm{a}}$.

\begin{tabular}{ll}
\hline \hline Property & Value \\
\hline Projected semimajor axis $^{\mathrm{b}}$ & $a \sin i=30.77 \pm 0.12 \mathrm{R}_{\odot}$ \\
Eccentricity $^{\mathrm{b} \text { c }}$ & $\mathrm{e}=0.47$ \\
Orbital period $^{\mathrm{b} \mathrm{c}}$ & $\mathrm{P}=13.609 \mathrm{~d}$ \\
Inclination $^{\mathrm{c}}$ & $i=65^{\circ}-70^{\circ}$ \\
Age $^{\mathrm{c}}$ & $10-30 \mathrm{Myrs}$ \\
Spectral type $^{\mathrm{b} \mathrm{c} \mathrm{d}}$ & $\mathrm{F} 5$ \\
Stellar Mass $^{\mathrm{c} \mathrm{d}}$ & $M_{*}=1.35 \pm 0.07 \mathrm{M}_{\odot}$ \\
Radius $^{\mathrm{c} \mathrm{d}}$ & $R_{*}=1.59 \pm 0.35 \mathrm{R}_{\odot}$ \\
Projected rotation velocity $^{\mathrm{bc} \mathrm{d}}$ & $v \sin i=18.5 \pm 1.0 \mathrm{~km} \mathrm{~s}^{-1}$ \\
\hline
\end{tabular}

${ }^{a}$ The ephemeris is $\tau(\min )=H J D 2446666.380+13.609 \times E$ from Andersen et al. 1989

${ }^{b}$ from Andersen et al. 1989

${ }^{c}$ from Alencar et al. 2003

${ }^{d}$ The system is composed by two nearly identical stars so these parameters are the same for both components. 